\title{
ON INTENSITY, MAGNETIC FIELD, AND VELOCITIES OF SMALL BRIGHT POINTS IN THE $\mathrm{CA}^{+}$NETWORK
}

\author{
DIRK SOLTAU \\ Kiepenheuer-Institut für Sonnenphysik \\ Schöneckstr. 6, D-7800 Freiburg, Germany
}

\begin{abstract}
More than 1000 small isolated bright $\mathrm{Ca}^{+}$structures (facular points) have been observed with the German VTT at the Observatorio del Teide, Tenerife. An analysis of Stokes V and Stokes I profiles of magnetically sensitive lines exhibit some typical properties of these features. The investigation is concentrated on those $40 \%$ of the observed structures for which the magnetic filling factor is larger than $10 \%$.
\end{abstract}

\section{INTRODUCTION}

$\mathrm{Ca}^{+} \mathrm{K}$ line emission is well correlated with magnetic flux and therefore bright Ca points are good tracers for magnetic flux concentrations. The nature of these structures which are also often called »facular points « or »calcium flocculi« is still not very well known. A statistical analysis of Stokes I and Stokes V spectra shall reveal information about the magnetic and temperature structure as well as the velocity behaviour of these features.

\section{OBSERVATIONS}

The observations have been done with the German Vacuum Tower Telescope on Tenerife. A superachromatic quarter wave plate and a polarizing beam splitter (calcite rod) just behind the spectrograph's entrance slit were used to record simultaneously $(\mathrm{I}+\mathrm{V})$ and (I-V) spectra on CCD cameras. The camera's pixel size was $0.1 \operatorname{arcsec} \times 2 \mathrm{~m} \AA$. 
By summing resp. subtracting those spectra one gets the Stokes I resp. Stokes V spectra. Such observations were made during three observing campaigns and more than 1000 targets have been observed. In most cases they coincide with the network boundary. Their position was chosen to cover the disk in a reasonable way. From these observations only those for which the maximum Stokes $\mathrm{V}$ amplitude was greater than $2 \%$ were considered in the following analysis. Such an amplitude corresponds to a filling factor of roughly $10 \%$. About $40 \%$ of the observations fulfill this condition.

For all these spectra a number of line parameters for the Stokes I as well as for the Stokes V profiles have been determined. The center of the structure was defined as the spatial point where a parabola fitted through the Stokes $V$ signal showed its maximum. The following results refer to this point.

\section{RESULTS}

\section{DIAMETER}

The full width at half maximum of the above mentioned parabola is a measure for the size of the magnetic structures. Figure 1 shows the measured size distribution for a sample at disc center. The peak at 2.5 arcsec is far beyond the resolution of the observations $(<1$ $\operatorname{arcsec})$. This indicates that there is a preference size for the observed magnetic structures.

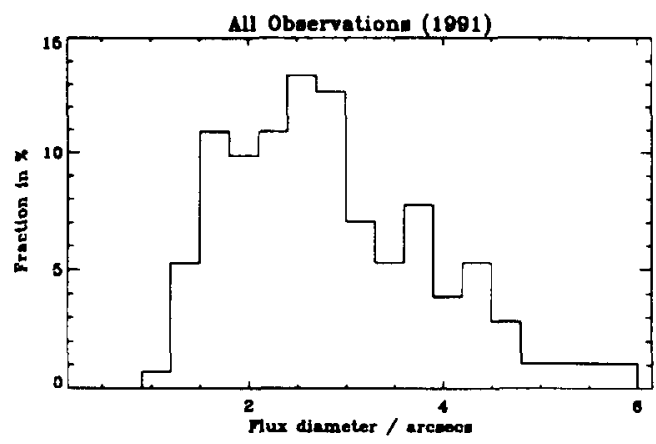

Fig.1 Measured size distribution for the spatial Stokes V profiles.

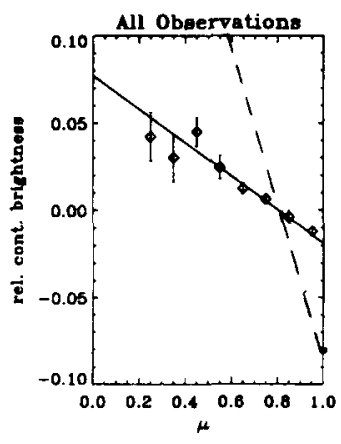

\section{INTENSITY}

For a long time the continuum contrast of bright $\mathrm{Ca}$ points was an open question. The large number of obsevations allowed for a statistical analysis and for a seeing correction of the measured continuum contrasts. Figure 2 shows the center-to-limb variation of the contrast. The dotted line corresponds to the seeing corrected values. At disc center the structures

Fig.2 Center-to-limb variation of continuum contrast. turn out to be dark $(-8 \%)$, at $\mu=0.8$ their contrast disap- 
pears and at the limb they appear bright $(+25 \%)$. This suggests that at deeper layers the magnetic elements are cooler (for equal optical depth). The explanation for the limb brightening may be not so easy, because here we possibly see not only a temperature excess in higher layers but also the »hot wall« effect.

\section{FIELD STRENGTH}

The observation of the wellknown line pair $\lambda 5247$ and $\lambda 5250$ allowed the application of the line ratio technique (Stenflo, 1973) to determine the intrinsic field strength of the targets. Figure 3 shows the normalized Stokes $\mathrm{V}$ amplitudes of the two lines plotted versus each other. The deviation from the

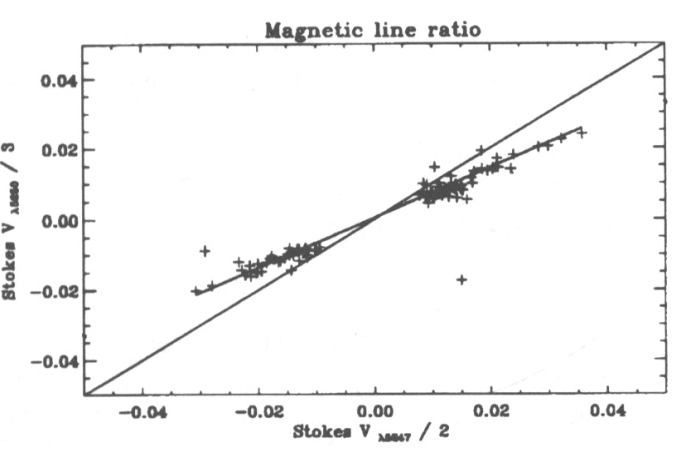

Fig. 3 Scatter plot for the normalized Stokes Amplitudes indicating the strong field character diagonal proves the strong field character. After calibration of the line ratio with a theoretical model (HSRA) the field strength is found to be 1400 Gauss.

\section{VELOCITIES}

Earlier investigations, e.g. Solanki (1986), excluded systematic material flows inside fluxtubes exceeding $250 \mathrm{~m} / \mathrm{s}$. This could be confirmed by the present work. But below this limit we found evidence for weak downflows of about $100 \mathrm{~m} / \mathrm{s}$ with respect to the immediate surrounding of the magnetic elements. For one particular structure a one hour

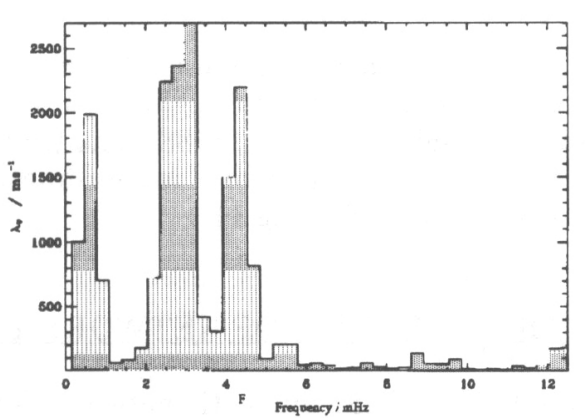

Fig. 4 Powerspectrum for Stokes V zerocrossing velocitiy of a selected $\mathrm{Ca}$ bright point time series has been observed which showed an oscillatory behaviour. Beside the p-mode peak at $3 \mathrm{mHz}$ the observation shows an additional peak at $4.5 \mathrm{mHz}$ (see Figure 4) which may give a hint for oscillatory motions in flux tubes.

\section{REFERENCES}

Stenflo, J.O. (1973): Solar Phys. 32, 41

Solanki, S.K. (1986): Astron. Astrophys. 168, 311 